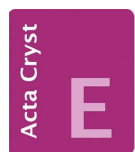

CRYSTALLOGRAPHIC COMMUNICATIONS

ISSN 2056-9890

\section{Crystal structure of an $\mathrm{Ag}^{\prime}$ intercalation compound: catena-poly[[silver(I)- $\mu$ - $N$-(pyridin-3-ylmethyl)- pyridin-3-amine- $\left.\kappa^{2} N: N^{\prime}\right]$ hexafluoridophosphate acetonitrile disolvate]}

\author{
Suk-Hee Moon, ${ }^{a}$ Youngjin Kang ${ }^{\mathrm{b} *}$ and Ki-Min Park ${ }^{\mathrm{c} *}$
}

Received 13 September 2017

Accepted 19 September 2017

Edited by $\mathrm{H}$. Stoeckli-Evans, University of Neuchâtel, Switzerland

Keywords: crystal structure; silver(I); dipyridyltype ligand; intercalation; Ag...Ag interaction; hydrogen bonding; $\pi-\pi$ interactions.

CCDC reference: 1575393

Supporting information: this article has supporting information at journals.iucr.org/e

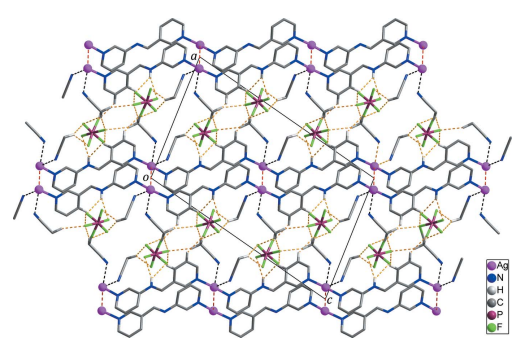

OPEN $\odot$ ACCESS
${ }^{\mathbf{a}}$ Department of Food and Nutrition, Kyungnam College of Information and Technology, Busan 47011 , Republic of Korea,
$\mathbf{b}^{\mathbf{b}}$ Division of Science Education, Kangwon National University, Chuncheon 24341, Republic of Korea, and ${ }^{\mathbf{c}}$ Research
institute of Natural Science, Gyeongsang National University, Jinju 52828, Republic of Korea. *Correspondence e-mail:
kangy@kangwon.ac.kr, kmpark@gnu.ac.kr

The asymmetric unit in the title compound, $\left[\mathrm{Ag}\left(\mathrm{C}_{11} \mathrm{H}_{11} \mathrm{~N}_{3}\right)\right] \mathrm{PF}_{6} \cdot 2 \mathrm{CH}_{3} \mathrm{CN}$ or $\left\{[\mathrm{Ag} L] \cdot \mathrm{PF}_{6} \cdot 2 \mathrm{CH}_{3} \mathrm{CN}\right\}_{n}, L=N$-(pyridin-3-ylmethyl)pyridin-3-amine, comprises one $\mathrm{Ag}^{\mathrm{I}}$ atom, one $L$ ligand, two acetonitrile solvent molecules and one $\mathrm{PF}_{6}{ }^{-}$ anion disordered over two orientations in a 0.567 (11):0.433 (11) ratio. Each $\mathrm{Ag}^{\mathrm{I}}$ atom is coordinated by two pyridine $\mathrm{N}$ atoms from two $L$ ligands in a slightly distorted linear coordination geometry $\left[\mathrm{N}-\mathrm{Ag}-\mathrm{N}=170.55(8)^{\circ}\right]$. Each $L$ ligand bridges two $\mathrm{Ag}^{\mathrm{I}}$ ions, resulting in the formation of a zigzag chain propagating along the [101] direction. In the crystal, Ag $\cdots A g$ contacts centroid distance $=3.5922(15) \AA]$ between the pyridine rings link these chains into a corrugated layer parallel to the (101) plane. The layers are stacked with a separation of 10.4532 (5) $\AA$, and acetonitrile solvent molecules and $\mathrm{PF}_{6}{ }^{-}$anions as guests are intercalated between the layers. The layers are connected through several $\mathrm{N} / \mathrm{C}-\mathrm{H} \cdots \mathrm{F}$ hydrogen bonds and $\mathrm{P}-\mathrm{F} \cdots \pi$ interactions $[\mathrm{F} \cdots$ ring centroid $=3.241(8) \AA$ ] between the layer and the intercalated guests and between the intercalated guests, forming a three-dimensional supramolecular network.

\section{Chemical context}

Silver coordination polymers based on dipyridyl-type ligands have been widely exploited due to the intriguing topologies and the fascinating properties caused by a variety of coordination geometries and $d^{10}$ electronic configurations of the $\mathrm{Ag}^{\mathrm{I}}$ ion (Leong \& Vittal, 2011; Moulton \& Zaworotko, 2001; Wang et al., 2012). In particular, $\mathrm{Ag}^{\mathrm{I}}$ ions have a preference for a linear two-coordinate geometry and can serve to link bridging dipyridyl-type ligands to form polymeric chains. Based on this concept, we have focused our attention on the development of one-dimensional $\mathrm{Ag}^{\mathrm{I}}$ coordination polymers with dipyridyltype ligands. Up to date, we have reported several $\mathrm{Ag}^{\mathrm{I}}$ coordination polymers with interesting topologies involving zigzag (Moon et al., 2016), helical (Moon et al., 2014, 2015) and double helical (Lee et al., 2015) structures. In an extension of our research, the title compound was prepared by the reaction of silver(I) hexafluoridophosphate with a dipyridyl typeligand, namely $N$-(pyridin-3-ylmethyl)pyridin-3-amine $(L)$, synthesized according to a literature procedure (Lee et al., 2013). Herein, we report on the crystal structure of the title compound in which lattice solvent molecules and anions as [3.3023 (5) $\AA$ ] and intermolecular $\pi-\pi$ stacking interactions [centroid-to- 
guests are intercalated between the layers formed by intermolecular interactions between zigzag $-(\mathrm{Ag}-L)_{n}-$ chains.

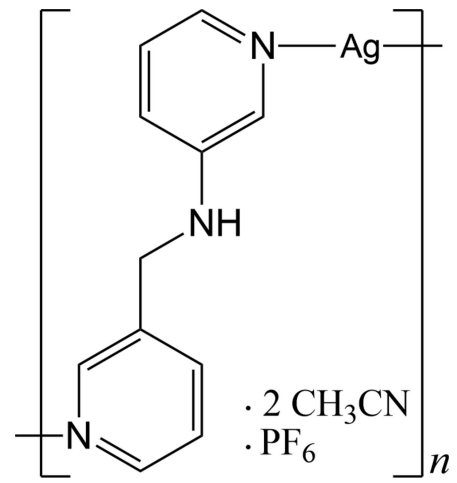

\section{Structural commentary}

The molecular components of the title structure are shown in Fig. 1. The asymmetric unit comprises one $\mathrm{Ag}^{\mathrm{I}}$ atom, one $L$ ligand, two acetonitrile solvent molecules, and one hexafluoridophosphate anion disordered over two orientations in a 0.567 (11):0.433 (11) ratio. The silver(I) atom is coordinated by two pyridine $\mathrm{N}$ atoms (N1 and N2) from two symmetryrelated $L$ ligands, leading to the formation of an infinite zigzag chain propagating along the [101] direction. Thus, the $\mathrm{Ag}^{\mathrm{I}}$ atom is two-coordinated in a slightly distorted linear coordination geometry $\left[\mathrm{N} 1{ }^{\mathrm{i}}-\mathrm{Ag} 1-\mathrm{N} 2=170.55(8)^{\circ}\right.$; symmetry code: (i) $x+\frac{1}{2},-y+\frac{1}{2}, z+\frac{1}{2}$; Table 1]. This distortion from linear geometry may be caused by Ag. . N interactions between the $\mathrm{Ag}^{\mathrm{I}}$ ion and two acetonitrile $\mathrm{N}$ atoms [Ag1 $\cdots \mathrm{N} 4=2.792(4)$, Ag1 $\cdots \mathrm{N} 5=2.815$ (4) A; black dashed lines in Fig. 1]. The two pyridine rings coordinated to the $\mathrm{Ag}^{\mathrm{I}}$ center are tilted slightly, by $6.29(15)^{\circ}$ with respect to each other. In the chain, the $\mathrm{Ag}^{\mathrm{I}}$ atoms are separated by 11.1009 (3) $\AA$ along the $L$ linker which

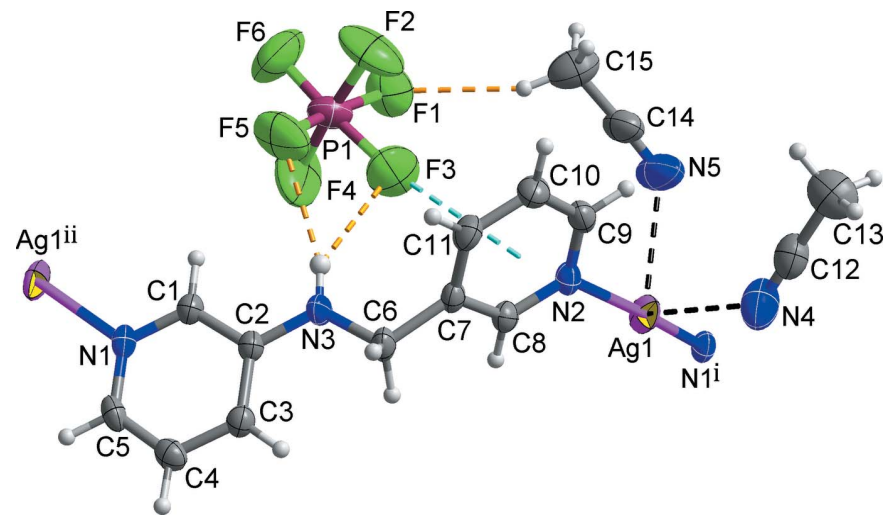

Figure 1

View of the molecular structure of the title compound, showing the atomnumbering scheme. Displacement ellipsoids are drawn at the $50 \%$ probability level. Disordered $\mathrm{F}$ atoms of the $\mathrm{PF}_{6}{ }^{-}$anion have been omitted for clarity. Black and yellow dashed lines represent $\mathrm{Ag} \cdots \mathrm{N}$ interactions and intermolecular $\mathrm{C} / \mathrm{N}-\mathrm{H} \cdots \mathrm{F}$ hydrogen bonds, respectively. [Symmetry codes: (i) $x+\frac{1}{2},-y+\frac{1}{2}, z+\frac{1}{2}$; (ii) $x-\frac{1}{2},-y+\frac{1}{2}, z-\frac{1}{2}$.]
Table 1

Selected geometric parameters $\left(\AA,{ }^{\circ}\right)$.

\begin{tabular}{lccc}
\hline $\mathrm{Ag} 1-\mathrm{N} 1{ }^{\mathrm{i}}$ & $2.163(2)$ & $\mathrm{Ag} 1-\mathrm{N} 2$ & $2.166(2)$ \\
$\mathrm{N} 1^{\mathrm{i}}-\mathrm{Ag} 1-\mathrm{N} 2$ & $170.55(8)$ & & \\
\hline
\end{tabular}

Symmetry code: (i) $x+\frac{1}{2},-y+\frac{1}{2}, z+\frac{1}{2}$.

Table 2

Hydrogen-bond geometry $\left(\AA{ }^{\circ}\right)$.

\begin{tabular}{lllll}
\hline$D-\mathrm{H} \cdots A$ & $D-\mathrm{H}$ & $\mathrm{H} \cdots A$ & $D \cdots A$ & $D-\mathrm{H} \cdots A$ \\
\hline $\mathrm{N} 3-\mathrm{H} 3 A \cdots \mathrm{F} 3$ & $0.84(3)$ & $2.49(3)$ & $3.208(8)$ & $144(3)$ \\
$\mathrm{N} 3-\mathrm{H} 3 A \cdots \mathrm{F} 5$ & $0.84(3)$ & $2.46(3)$ & $3.273(10)$ & $162(3)$ \\
$\mathrm{N} 3-\mathrm{H} 3 A \cdots \mathrm{F} 5^{\prime}$ & $0.84(3)$ & $2.18(3)$ & $2.999(10)$ & $167(3)$ \\
$\mathrm{C} 8-\mathrm{H} 8 \cdots 4^{\mathrm{ii}}$ & 0.95 & 2.63 & $3.478(5)$ & 149 \\
$\mathrm{C} 10-\mathrm{H} 10 \cdots \mathrm{F}^{\mathrm{iii}}$ & 0.95 & 2.39 & $3.176(9)$ & 140 \\
$\mathrm{C} 13-\mathrm{H} 13 A \cdots \mathrm{F} 2^{\text {iii }}$ & 0.98 & 2.54 & $3.487(13)$ & 163 \\
$\mathrm{C} 13-\mathrm{H} 13 B \cdots \mathrm{F} 6^{\mathrm{i}}$ & 0.98 & 2.53 & $3.365(10)$ & 144 \\
$\mathrm{C} 13-\mathrm{H} 13 B \cdots \mathrm{F} 1^{\text {ii }}$ & 0.98 & 2.59 & $3.57(2)$ & 171 \\
$\mathrm{C} 15-\mathrm{H} 15 A \cdots \mathrm{F} 1$ & 0.98 & 2.52 & $3.441(9)$ & 157 \\
$\mathrm{C} 15-\mathrm{H} 15 A \cdots \mathrm{F} 3^{\prime}$ & 0.98 & 2.42 & $3.356(13)$ & 160 \\
$\mathrm{C} 15-\mathrm{H} 15 B \cdots 2^{\text {iv }}$ & 0.98 & 2.23 & $3.088(10)$ & 146 \\
$\mathrm{C} 15-\mathrm{H} 15 B \cdots 5^{\text {iv }}$ & 0.98 & 2.57 & $3.509(11)$ & 160 \\
$\mathrm{C} 15-\mathrm{H} 15 C \cdots \mathrm{F}^{\text {iii }}$ & 0.98 & 2.36 & $3.329(8)$ & 168 \\
$\mathrm{C} 15-\mathrm{H} 15 C \cdots 1^{\text {iii }}$ & 0.98 & 2.09 & $3.037(9)$ & 161
\end{tabular}

Symmetry codes: (i) $\quad x+\frac{1}{2},-y+\frac{1}{2}, z+\frac{1}{2} ; \quad$ (ii) $\quad-x+1,-y+1,-z+1$; $\quad$ (iii) $-x+\frac{3}{2}, y+\frac{1}{2},-z+\frac{1}{2}$; (iv) $-x+\frac{3}{2}, y-\frac{1}{2},-z+\frac{1}{2}$.

adopts a stretched trans conformation with the $\mathrm{C} 2-\mathrm{N} 3-\mathrm{C} 6-$ C7 torsion angles being $174.7(3)^{\circ}$.

\section{Supramolecular features}

The neighbouring zigzag chains are connected by Ag...Ag

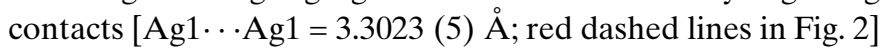
and intermolecular $\pi$ - $\pi$-stacking interactions between the pyridine rings $\left[C g 1 \cdots C g 2^{\mathrm{ii}}=3.5922(15) \AA\right.$; yellow dashed lines in Fig. 2; $C g 1$ and $C g 2$ are the centroids of the N1/C1-C5 and N2/C7-C11 rings, respectively; symmetry code: (ii) $-x+\frac{1}{2}$, $y-\frac{1}{2},-z+\frac{1}{2}$ ], resulting in the formation of a corrugated layer spreading out along the (101) plane (Fig. 2). Adjacent layers are stacked on each other with a separation of 10.4532 (5) $\AA$. Acetonitrile molecules and $\mathrm{PF}_{6}{ }^{-}$anions as guests are intercalated between the layers (Fig. 3). The layers are further

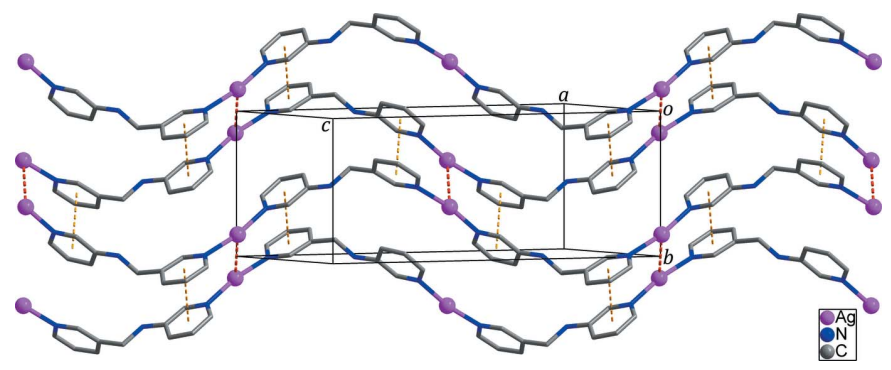

Figure 2

The two-dimensional network formed through $\mathrm{Ag} \cdots \mathrm{Ag}$ contacts (red dashed lines) and intermolecular $\pi-\pi$ stacking interactions (yellow dashed lines). Acetonitrile solvent molecules, the $\mathrm{PF}_{6}{ }^{-}$anions and $\mathrm{H}$ atoms have been omitted for clarity. 


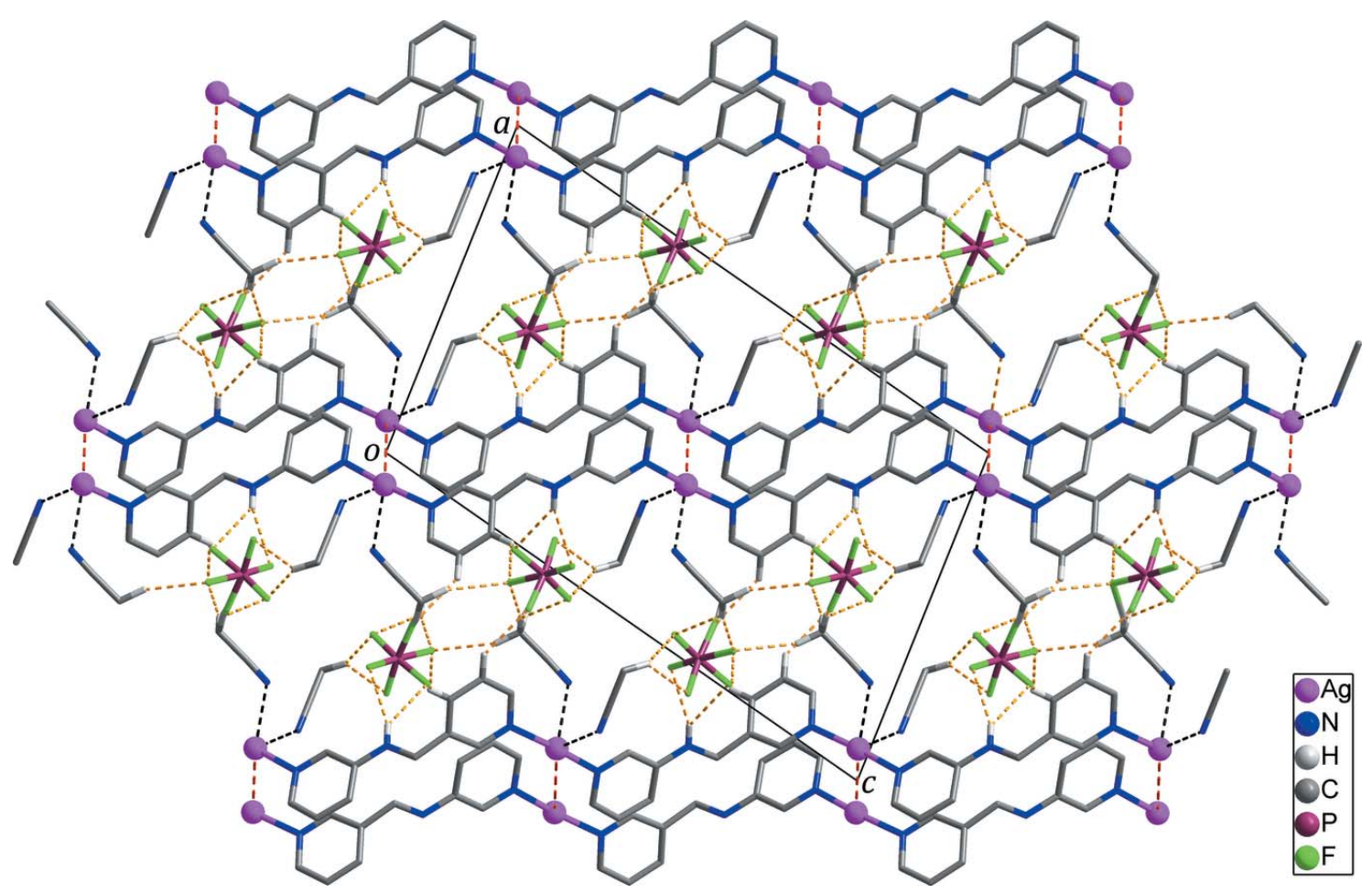

Figure 3

Interlayer stacking showing the intercalation of acetonitrile molecules and $\mathrm{PF}_{6}{ }^{-}$anions between the layers. Red, black and yellow dashed lines represent Ag $\cdots$ Ag contacts, Ag $\cdots \mathrm{N}$ interactions and $\mathrm{N} / \mathrm{C}-\mathrm{H} \cdots \mathrm{F}$ hydrogen bonds, respectively. Disordered $\mathrm{F}$ atoms of the $\mathrm{PF}_{6}{ }^{-}$anions and $\mathrm{H}$ atoms not involved in intermolecular interactions have been omitted for clarity.

Table 3

Experimental details.

\begin{tabular}{|c|c|}
\hline Crystal data & \\
\hline Chemical formula & {$\left[\mathrm{Ag}\left(\mathrm{C}_{11} \mathrm{H}_{11} \mathrm{~N}_{3}\right)\right] \mathrm{PF}_{6} \cdot 2 \mathrm{C}_{2} \mathrm{H}_{3} \mathrm{~N}$} \\
\hline$M_{\mathrm{r}}$ & 520.17 \\
\hline Crystal system, space group & Monoclinic, $P 2_{1} / n$ \\
\hline Temperature (K) & 173 \\
\hline$a, b, c(\AA)$ & $12.8997(4), 7.5361(3), 20.9747$ (7) \\
\hline$\beta\left(^{\circ}\right)$ & $102.9900(6)$ \\
\hline$V\left(\AA^{3}\right)$ & $1986.84(12)$ \\
\hline$Z$ & 4 \\
\hline Radiation type & Mo $K \alpha$ \\
\hline$\mu\left(\mathrm{mm}^{-1}\right)$ & 1.16 \\
\hline Crystal size $(\mathrm{mm})$ & $0.35 \times 0.25 \times 0.15$ \\
\hline Data collection & \\
\hline Diffractometer & Bruker APEXII CCD \\
\hline Absorption correction & $\begin{array}{l}\text { Multi-scan (SADABS; Bruker, } \\
\text { 2014) }\end{array}$ \\
\hline$T_{\min }, T_{\max }$ & $0.666,0.746$ \\
\hline $\begin{array}{l}\text { No. of measured, independent and } \\
\text { observed }[I>2 \sigma(I)] \text { reflections }\end{array}$ & $11824,4316,3527$ \\
\hline$R_{\text {int }}$ & 0.020 \\
\hline$(\sin \theta / \lambda)_{\max }\left(\AA^{-1}\right)$ & 0.639 \\
\hline Refinement & \\
\hline$R\left[F^{2}>2 \sigma\left(F^{2}\right)\right], w R\left(F^{2}\right), S$ & $0.031,0.081,1.10$ \\
\hline No. of reflections & 4316 \\
\hline No. of parameters & 312 \\
\hline No. of restraints & 18 \\
\hline $\mathrm{H}$-atom treatment & $\begin{array}{l}\mathrm{H} \text { atoms treated by a mixture of } \\
\text { independent and constrained } \\
\text { refinement }\end{array}$ \\
\hline$\Delta \rho_{\max }, \Delta \rho_{\min }\left(\mathrm{e} \AA^{-3}\right)$ & $1.12,-0.66$ \\
\hline
\end{tabular}

Computer programs: APEX2 and SAINT (Bruker, 2014), SHELXS97 and SHELXTL (Sheldrick, 2008), SHELXL2014 (Sheldrick, 2015), DIAMOND (Brandenburg, 2010) and publCIF (Westrip, 2010). connected by several intermolecular $\mathrm{N} / \mathrm{C}-\mathrm{H} \cdots \mathrm{F}$ hydrogen bonds (Table 2; yellow dashed lines in Figs. 1 and 3) and $\mathrm{P}-$ $\mathrm{F} \cdots \pi$ interactions $[\mathrm{F} 3 \cdots C g 2=3.241(8) \AA$; sky-blue dashed line in Fig. 1] between the layer and the anions and between the acetonitrile solvent molecules and the anions, forming a three-dimensional supramolecular network.

\section{Synthesis and crystallization}

The $L$ ligand was synthesized according to a literature method (Lee et al., 2013). Slow evaporation of an acetonitrile solution of the $L$ ligand with $\mathrm{AgPF}_{6}$ in the molar ratio 1:1 afforded colourless block-like X-ray quality single crystals of the title compound.

\section{Refinement}

Crystal data, data collection and structure refinement details are summarized in Table 3. The $\mathrm{PF}_{6}{ }^{-}$anion is disordered over two orientations in a 0.567 (11):0.433 (11) ratio. The amine $\mathrm{H}$ atom was located from a difference-Fourier map and freely refined $[\mathrm{N}-\mathrm{H}=0.84(3) \AA]$. All other $\mathrm{H}$ atoms were positioned geometrically and refined as riding: $\mathrm{C}-\mathrm{H}=0.95 \AA$ for $\mathrm{Csp}{ }^{2}-\mathrm{H}, 0.99 \AA$ for methylene $\mathrm{C}-\mathrm{H}$ and $0.98 \AA$ for methyl $\mathrm{C}-\mathrm{H}$ with $U_{\text {iso }}(\mathrm{H})=1.5 U_{\text {eq }}(\mathrm{C}$-methyl $)$ and $1.2 U_{\text {eq }}(\mathrm{C})$ for other $\mathrm{C}$-bound $\mathrm{H}$ atoms. 


\section{Funding information}

This research was supported by the Basic Science Research Program through the National Research Foundation of Korea (NRF) funded by the Ministry of Education (NRF2015R1D1A3A01020410 and NRF-2016R1D1A1B01012630).

\section{References}

Brandenburg, K. (2010). DIAMOND. Crystal Impact GbR, Bonn, Germany.

Bruker (2014). APEX2, SAINT and SADABS. Bruker AXS Inc., Madison, Wisconsin, USA.

Lee, E., Ju, H., Moon, S.-H., Lee, S. S. \& Park, K.-M. (2015). Bull. Korean Chem. Soc. 36, 1532-1535.
Lee, E., Ryu, H., Moon, S.-H. \& Park, K.-M. (2013). Bull. Korean Chem. Soc. 34, 3477-3480.

Leong, W. L. \& Vittal, J. J. (2011). Chem. Rev. 111, 688-764.

Moon, B., Jeon, Y., Moon, S.-H. \& Park, K.-M. (2014). Acta Cryst. E70, 507-509.

Moon, S.-H., Kang, Y. \& Park, K.-M. (2015). Acta Cryst. E71, 12871289.

Moon, S.-H., Kang, D. \& Park, K.-M. (2016). Acta Cryst. E72, 15131516.

Moulton, B. \& Zaworotko, M. J. (2001). Chem. Rev. 101, 16291658.

Sheldrick, G. M. (2008). Acta Cryst. A64, 112-122.

Sheldrick, G. M. (2015). Acta Cryst. C71, 3-8.

Wang, C., Zhang, T. \& Lin, W. (2012). Chem. Rev. 112, 1084-1104.

Westrip, S. P. (2010). J. Appl. Cryst. 43, 920-925. 


\section{supporting information}

Acta Cryst. (2017). E73, 1542-1545 [https://doi.org/10.1107/S2056989017013421]

\section{Crystal structure of an Ag' intercalation compound: catena-poly[[silver(I)- $\mu$ -} $N$-(pyridin-3-ylmethyl) pyridin-3-amine- $\left.\kappa^{2} N: N^{\prime}\right]$ hexafluoridophosphate acetonitrile disolvate]

\section{Suk-Hee Moon, Youngjin Kang and Ki-Min Park}

Computing details

Data collection: APEX2 (Bruker, 2014); cell refinement: SAINT (Bruker, 2014); data reduction: SAINT (Bruker, 2014); program(s) used to solve structure: SHELXS97 (Sheldrick, 2008); program(s) used to refine structure: SHELXL2014 (Sheldrick, 2015); molecular graphics: DIAMOND (Brandenburg, 2010); software used to prepare material for publication: SHELXTL (Sheldrick, 2008) and publCIF (Westrip, 2010).

catena-Poly[[silver(I)- $\mu$ - $N$-(pyridin-3-ylmethyl) pyridine-3-amine- $\left.\kappa^{2} N^{\prime} N^{\prime}\right]$ hexafluoridophosphate acetonitrile disolvate]

Crystal data

$\left[\mathrm{Ag}\left(\mathrm{C}_{11} \mathrm{H}_{11} \mathrm{~N}_{3}\right)\right] \mathrm{PF}_{6} \cdot 2 \mathrm{C}_{2} \mathrm{H}_{3} \mathrm{~N}$

$M_{r}=520.17$

Monoclinic, $P 2_{1} / n$

$a=12.8997$ (4) $\AA$

$b=7.5361(3) \AA$

$c=20.9747(7) \AA$

$\beta=102.9900(6)^{\circ}$

$V=1986.84(12) \AA^{3}$

$Z=4$

\section{Data collection}

\section{Bruker APEXII CCD}

diffractometer

$\varphi$ and $\omega$ scans

Absorption correction: multi-scan

(SADABS; Bruker, 2014)

$T_{\min }=0.666, T_{\max }=0.746$

11824 measured reflections

\section{Refinement}

Refinement on $F^{2}$

Least-squares matrix: full

$R\left[F^{2}>2 \sigma\left(F^{2}\right)\right]=0.031$

$w R\left(F^{2}\right)=0.081$

$S=1.10$

4316 reflections

312 parameters

18 restraints
$F(000)=1032$

$D_{\mathrm{x}}=1.739 \mathrm{Mg} \mathrm{m}^{-3}$

Mo $K \alpha$ radiation, $\lambda=0.71073 \AA$

Cell parameters from 5825 reflections

$\theta=2.9-28.0^{\circ}$

$\mu=1.16 \mathrm{~mm}^{-1}$

$T=173 \mathrm{~K}$

Block, colorless

$0.35 \times 0.25 \times 0.15 \mathrm{~mm}$

4316 independent reflections

3527 reflections with $I>2 \sigma(I)$

$R_{\text {int }}=0.020$

$\theta_{\text {max }}=27.0^{\circ}, \theta_{\min }=1.7^{\circ}$

$h=-10 \rightarrow 16$

$k=-9 \rightarrow 9$

$l=-26 \rightarrow 26$

Primary atom site location: structure-invariant direct methods

Secondary atom site location: difference Fourier map

Hydrogen site location: mixed

$\mathrm{H}$ atoms treated by a mixture of independent and constrained refinement 
$w=1 /\left[\sigma^{2}\left(F_{\mathrm{o}}^{2}\right)+(0.0297 P)^{2}+2.2488 P\right]$

where $P=\left(F_{\mathrm{o}}^{2}+2 F_{\mathrm{c}}{ }^{2}\right) / 3$

$(\Delta / \sigma)_{\max }=0.001$

$$
\Delta \rho_{\max }=1.12 \mathrm{e} \AA^{-3}
$$

$\Delta \rho_{\min }=-0.66 \mathrm{e} \AA^{-3}$

\section{Special details}

Geometry. All esds (except the esd in the dihedral angle between two 1.s. planes) are estimated using the full covariance matrix. The cell esds are taken into account individually in the estimation of esds in distances, angles and torsion angles; correlations between esds in cell parameters are only used when they are defined by crystal symmetry. An approximate (isotropic) treatment of cell esds is used for estimating esds involving 1.s. planes.

Fractional atomic coordinates and isotropic or equivalent isotropic displacement parameters $\left(\AA^{2}\right)$

\begin{tabular}{|c|c|c|c|c|c|}
\hline & $x$ & $y$ & $z$ & $U_{\text {iso }} * / U_{\text {eq }}$ & Occ. $(<1)$ \\
\hline Ag1 & $0.57809(2)$ & 0.34509 & $0.48065(2)$ & $0.04382(9)$ & \\
\hline N1 & $0.09170(18)$ & $0.3315(3)$ & $0.06343(10)$ & $0.0313(5)$ & \\
\hline $\mathrm{N} 2$ & $0.55270(18)$ & $0.4849(3)$ & $0.38820(10)$ & $0.0317(5)$ & \\
\hline N3 & $0.3023(2)$ & $0.4904(4)$ & $0.19724(12)$ & $0.0385(6)$ & \\
\hline $\mathrm{H} 3 \mathrm{~A}$ & $0.352(3)$ & $0.439(4)$ & $0.1850(15)$ & $0.035(8) *$ & \\
\hline $\mathrm{C} 1$ & $0.1875(2)$ & $0.3614(4)$ & $0.10237(12)$ & $0.0305(6)$ & \\
\hline H1 & 0.2478 & 0.3053 & 0.0924 & $0.037 *$ & \\
\hline $\mathrm{C} 2$ & $0.2022(2)$ & $0.4724(3)$ & $0.15729(12)$ & $0.0282(5)$ & \\
\hline $\mathrm{C} 3$ & $0.1127(2)$ & $0.5557(4)$ & $0.17056(13)$ & $0.0315(6)$ & \\
\hline $\mathrm{H} 3$ & 0.1192 & 0.6331 & 0.2070 & $0.038^{*}$ & \\
\hline $\mathrm{C} 4$ & $0.0141(2)$ & 0.5233 & $0.12960(13)$ & $0.0347(6)$ & \\
\hline $\mathrm{H} 4$ & -0.0477 & 0.5784 & 0.1379 & $0.042 *$ & \\
\hline $\mathrm{C} 5$ & $0.0059(2)$ & $0.4111(4)$ & $0.07669(13)$ & $0.0328(6)$ & \\
\hline H5 & -0.0620 & 0.3897 & 0.0490 & $0.039 *$ & \\
\hline C6 & $0.3223(2)$ & $0.6106(4)$ & $0.25170(13)$ & $0.0363(6)$ & \\
\hline H6A & 0.2721 & 0.5853 & 0.2800 & $0.044 *$ & \\
\hline H6B & 0.3097 & 0.7338 & 0.2354 & $0.044^{*}$ & \\
\hline $\mathrm{C} 7$ & $0.4343(2)$ & $0.5940(3)$ & $0.29117(12)$ & $0.0288(5)$ & \\
\hline $\mathrm{C} 8$ & $0.4549(2)$ & $0.5027(3)$ & $0.34988(12)$ & $0.0294(5)$ & \\
\hline $\mathrm{H} 8$ & 0.3968 & 0.4500 & 0.3638 & $0.035^{*}$ & \\
\hline C9 & $0.6343(2)$ & $0.5596(4)$ & $0.36808(14)$ & $0.0355(6)$ & \\
\hline $\mathrm{H} 9$ & 0.7041 & 0.5474 & 0.3946 & $0.043^{*}$ & \\
\hline $\mathrm{C} 10$ & $0.6207(2)$ & $0.6531(4)$ & $0.31048(14)$ & $0.0400(7)$ & \\
\hline $\mathrm{H} 10$ & 0.6801 & 0.7041 & 0.2976 & $0.048^{*}$ & \\
\hline $\mathrm{C} 11$ & $0.5193(2)$ & $0.6720(4)$ & $0.27147(13)$ & $0.0374(6)$ & \\
\hline H11 & 0.5082 & 0.7374 & 0.2318 & $0.045 *$ & \\
\hline $\mathrm{P} 1$ & $0.52792(8)$ & $0.16310(12)$ & $0.15620(5)$ & $0.0532(2)$ & \\
\hline $\mathrm{F} 1$ & $0.6049(5)$ & $0.0241(8)$ & 0.2082 & 0.0812 (19) & $0.567(11)$ \\
\hline $\mathrm{F} 2$ & $0.6355(6)$ & $0.2633(12)$ & $0.1590(6)$ & $0.106(3)$ & $0.567(11)$ \\
\hline F3 & $0.5178(6)$ & $0.2625(10)$ & 0.2228 & $0.106(3)$ & $0.567(11)$ \\
\hline F4 & $0.4273(13)$ & $0.060(3)$ & $0.1615(8)$ & $0.133(6)$ & $0.567(11)$ \\
\hline F5 & $0.4615(6)$ & $0.3036(14)$ & $0.1156(4)$ & $0.100(3)$ & $0.567(11)$ \\
\hline F6 & $0.5453(8)$ & $0.0508(11)$ & $0.0998(4)$ & $0.119(3)$ & $0.567(11)$ \\
\hline $\mathrm{F} 1^{\prime}$ & $0.5583(12)$ & $-0.0286(12)$ & $0.1587(9)$ & $0.149(7)$ & $0.433(11)$ \\
\hline $\mathrm{F} 2^{\prime}$ & $0.6253(9)$ & $0.2192(19)$ & $0.1252(6)$ & $0.106(4)$ & $0.433(11)$ \\
\hline F3' & $0.5773(14)$ & $0.211(2)$ & $0.2223(5)$ & $0.184(7)$ & $0.433(11)$ \\
\hline
\end{tabular}




$\begin{array}{llllll}\text { F4' }^{\prime} & 0.4247(14) & 0.101(3) & 0.1739(9) & 0.128(7) & 0.433(11) \\ \text { F5' }^{\prime} & 0.4867(8) & 0.3671(11) & 0.1435(6) & 0.086(3) & 0.433(11) \\ \text { F6' } & 0.4650(11) & 0.1392(17) & 0.0770(4) & 0.125(5) & 0.433(11) \\ \text { N4 } & 0.6922(3) & 0.6394(5) & 0.53962(16) & 0.0733(10) & 0.0538(9) \\ \text { C12 } & 0.7722(3) & 0.6996(5) & 0.53960(16) & 0.0756(12) \\ \text { C13 } & 0.8750(3) & 0.7783(6) & 0.5397(2) & 0.113^{*} \\ \text { H13A } & 0.8918 & 0.7604 & 0.4969 & 0.113^{*} \\ \text { H13B } & 0.9298 & 0.7219 & 0.5736 & 0.113^{*} \\ \text { H13C } & 0.8727 & 0.9057 & 0.5487 & 0.0922(14) \\ \text { N5 } & 0.7553(3) & 0.1622(7) & 0.45595(18) & 0.0566(9) \\ \text { C14 } & 0.7749(3) & 0.1581(5) & 0.40711(19) & 0.0749(12) \\ \text { C15 } & 0.7958(4) & 0.1556(6) & 0.3423(2) & 0.112^{*} \\ \text { H15A } & 0.7287 & 0.1410 & 0.3098 & 0.112^{*} \\ \text { H15B } & 0.8435 & 0.0566 & 0.3388 & 0.112^{*} \\ \text { H15C } & 0.8295 & 0.2675 & 0.3343 & \end{array}$

Atomic displacement parameters $\left(\AA^{2}\right)$

\begin{tabular}{|c|c|c|c|c|c|c|}
\hline & $U^{11}$ & $U^{22}$ & $U^{33}$ & $U^{12}$ & $U^{13}$ & $U^{23}$ \\
\hline Ag1 & $0.05358(16)$ & $0.04805(15)$ & $0.02636(12)$ & $0.01328(11)$ & $0.00167(9)$ & $0.01001(10)$ \\
\hline N1 & $0.0354(12)$ & $0.0327(12)$ & $0.0226(10)$ & $-0.0052(10)$ & $-0.0003(9)$ & $0.0008(9)$ \\
\hline N2 & $0.0343(12)$ & $0.0324(12)$ & $0.0246(11)$ & $0.0031(10)$ & $-0.0010(9)$ & $0.0005(9)$ \\
\hline N3 & $0.0288(12)$ & $0.0498(15)$ & $0.0335(12)$ & $0.0031(11)$ & $0.0000(10)$ & $-0.0170(11)$ \\
\hline $\mathrm{C} 1$ & 0.0309 (14) & 0.0348 (14) & $0.0245(12)$ & $0.0000(11)$ & $0.0037(10)$ & $-0.0022(10)$ \\
\hline $\mathrm{C} 2$ & $0.0296(13)$ & 0.0307 (13) & $0.0227(12)$ & $-0.0005(11)$ & $0.0026(10)$ & $0.0000(10)$ \\
\hline $\mathrm{C} 3$ & $0.0349(14)$ & $0.0309(14)$ & $0.0273(13)$ & $0.0024(11)$ & $0.0039(11)$ & $-0.0011(10)$ \\
\hline $\mathrm{C} 4$ & $0.0310(14)$ & $0.0341(14)$ & $0.0373(15)$ & 0.0061 (11) & $0.0040(11)$ & $0.0072(12)$ \\
\hline $\mathrm{C} 5$ & $0.0276(13)$ & $0.0356(14)$ & $0.0295(13)$ & $-0.0035(11)$ & $-0.0055(10)$ & $0.0081(11)$ \\
\hline C6 & $0.0337(15)$ & $0.0436(16)$ & $0.0268(13)$ & $0.0037(12)$ & $-0.0034(11)$ & $-0.0095(11)$ \\
\hline $\mathrm{C} 7$ & $0.0325(14)$ & $0.0291(13)$ & $0.0221(12)$ & $0.0020(11)$ & $0.0005(10)$ & $-0.0053(10)$ \\
\hline $\mathrm{C} 8$ & $0.0316(14)$ & $0.0283(13)$ & $0.0273(12)$ & $-0.0023(11)$ & $0.0042(10)$ & $-0.0036(10)$ \\
\hline C9 & $0.0292(14)$ & $0.0393(16)$ & $0.0347(14)$ & $0.0019(12)$ & 0.0005 (11) & $-0.0060(12)$ \\
\hline $\mathrm{C} 10$ & $0.0355(15)$ & $0.0484(17)$ & $0.0369(15)$ & $-0.0087(13)$ & $0.0101(12)$ & $-0.0023(13)$ \\
\hline $\mathrm{C} 11$ & $0.0451(16)$ & $0.0419(16)$ & $0.0246(13)$ & $-0.0021(13)$ & $0.0066(11)$ & $0.0027(11)$ \\
\hline $\mathrm{P} 1$ & $0.0471(5)$ & $0.0444(5)$ & $0.0736(6)$ & $0.0043(4)$ & $0.0254(5)$ & $-0.0012(4)$ \\
\hline $\mathrm{F} 1$ & $0.086(4)$ & $0.064(3)$ & $0.087(4)$ & $0.018(3)$ & $0.007(3)$ & $0.016(3)$ \\
\hline $\mathrm{F} 2$ & $0.052(3)$ & $0.092(5)$ & $0.175(8)$ & -0.015 & $0.027(5)$ & $0.043(5)$ \\
\hline F3 & $0.103(5)$ & $0.094(4)$ & $0.119(5)$ & $0.022(4)$ & $0.022(4)$ & -0.057 (4) \\
\hline F4 & $0.115(10)$ & $0.173(10)$ & $0.102(7)$ & $-0.101(9)$ & $0.007(6)$ & $-0.010(6)$ \\
\hline F5 & $0.069(4)$ & $0.118(6)$ & $0.110(6)$ & $0.038(4)$ & $0.014(4)$ & $0.051(5)$ \\
\hline F6 & $0.159(7)$ & $0.143(7)$ & $0.065(4)$ & $0.024(6)$ & $0.044(4)$ & $-0.022(4)$ \\
\hline $\mathrm{F} 1^{\prime}$ & $0.171(11)$ & $0.056(5)$ & $0.24(2)$ & $0.039(6)$ & $0.097(12)$ & $0.046(7)$ \\
\hline $\mathrm{F}^{\prime}$ & $0.049(5)$ & $0.153(10)$ & $0.123(8)$ & $0.030(6)$ & $0.036(5)$ & $0.007(7)$ \\
\hline $\mathrm{F}^{\prime}$ & $0.183(14)$ & $0.256(18)$ & $0.076(7)$ & $0.038(13)$ & $-0.048(8)$ & $-0.028(8)$ \\
\hline $\mathrm{F} 4^{\prime}$ & $0.083(9)$ & $0.215(18)$ & $0.105(10)$ & $-0.012(9)$ & $0.063(8)$ & $0.054(10)$ \\
\hline $\mathrm{F}^{\prime}$ & $0.077(5)$ & $0.063(4)$ & $0.129(7)$ & $0.016(3)$ & $0.047(5)$ & -0.005 (4) \\
\hline F6 ${ }^{\prime}$ & $0.154(10)$ & $0.152(10)$ & $0.059(4)$ & $-0.049(8)$ & $0.002(5)$ & $-0.006(5)$ \\
\hline N4 & $0.084(3)$ & $0.080(3)$ & $0.0521(19)$ & $-0.026(2)$ & 0.0085 (17) & $-0.0043(17)$ \\
\hline
\end{tabular}




\begin{tabular}{lllllll}
$\mathrm{C} 12$ & $0.067(2)$ & $0.051(2)$ & $0.0397(18)$ & $-0.0057(18)$ & $0.0052(16)$ & $-0.0009(15)$ \\
$\mathrm{C} 13$ & $0.068(3)$ & $0.068(3)$ & $0.088(3)$ & $-0.002(2)$ & $0.013(2)$ & $0.013(2)$ \\
$\mathrm{N} 5$ & $0.067(2)$ & $0.156(4)$ & $0.057(2)$ & $0.044(3)$ & $0.0217(18)$ & $0.025(2)$ \\
C14 & $0.0406(18)$ & $0.071(2)$ & $0.060(2)$ & $0.0125(17)$ & $0.0159(16)$ & $0.0091(19)$ \\
C15 & $0.092(3)$ & $0.071(3)$ & $0.074(3)$ & $-0.001(2)$ & $0.044(3)$ & $0.003(2)$ \\
\hline
\end{tabular}

Geometric parameters $\left(\hat{A},{ }^{\circ}\right)$

\begin{tabular}{|c|c|c|c|}
\hline $\mathrm{Ag} 1-\mathrm{N} 1^{\mathrm{i}}$ & $2.163(2)$ & C9-H9 & 0.9500 \\
\hline $\mathrm{Ag} 1-\mathrm{N} 2$ & $2.166(2)$ & $\mathrm{C} 10-\mathrm{C} 11$ & $1.385(4)$ \\
\hline $\mathrm{Ag} 1-\mathrm{Ag} 1^{\mathrm{ii}}$ & $3.3023(5)$ & $\mathrm{C} 10-\mathrm{H} 10$ & 0.9500 \\
\hline $\mathrm{N} 1-\mathrm{C} 1$ & $1.338(3)$ & $\mathrm{C} 11-\mathrm{H} 11$ & 0.9500 \\
\hline $\mathrm{N} 1-\mathrm{C} 5$ & $1.341(4)$ & $\mathrm{P} 1-\mathrm{F} 3^{\prime}$ & $1.435(10)$ \\
\hline $\mathrm{N} 1-\mathrm{Ag} 1^{\mathrm{iii}}$ & $2.163(2)$ & $\mathrm{P} 1-\mathrm{F} 1^{\prime}$ & $1.495(8)$ \\
\hline $\mathrm{N} 2-\mathrm{C} 8$ & $1.342(3)$ & $\mathrm{P} 1-\mathrm{F} 5$ & $1.501(6)$ \\
\hline $\mathrm{N} 2-\mathrm{C} 9$ & $1.343(4)$ & $\mathrm{P} 1-\mathrm{F} 6$ & $1.512(5)$ \\
\hline $\mathrm{N} 3-\mathrm{C} 2$ & $1.379(3)$ & $\mathrm{P} 1-\mathrm{F} 4^{\prime}$ & $1.532(14)$ \\
\hline $\mathrm{N} 3-\mathrm{C} 6$ & $1.435(3)$ & $\mathrm{P} 1-\mathrm{F} 4$ & $1.539(12)$ \\
\hline $\mathrm{N} 3-\mathrm{H} 3 \mathrm{~A}$ & $0.84(3)$ & $\mathrm{P} 1-\mathrm{F} 2$ & $1.570(7)$ \\
\hline $\mathrm{C} 1-\mathrm{C} 2$ & $1.401(3)$ & $\mathrm{P} 1-\mathrm{F} 2^{\prime}$ & $1.596(11)$ \\
\hline $\mathrm{C} 1-\mathrm{H} 1$ & 0.9500 & $\mathrm{P} 1-\mathrm{F} 3$ & $1.617(6)$ \\
\hline $\mathrm{C} 2-\mathrm{C} 3$ & $1.396(4)$ & $\mathrm{P} 1-\mathrm{F} 5^{\prime}$ & $1.629(9)$ \\
\hline $\mathrm{C} 3-\mathrm{C} 4$ & $1.387(4)$ & $\mathrm{P} 1-\mathrm{F} 1$ & $1.669(5)$ \\
\hline $\mathrm{C} 3-\mathrm{H} 3$ & 0.9500 & $\mathrm{P} 1-\mathrm{F} 6^{\prime}$ & $1.687(8)$ \\
\hline $\mathrm{C} 4-\mathrm{C} 5$ & $1.380(4)$ & $\mathrm{N} 4-\mathrm{C} 12$ & $1.127(5)$ \\
\hline $\mathrm{C} 4-\mathrm{H} 4$ & 0.9500 & $\mathrm{C} 12-\mathrm{C} 13$ & $1.452(6)$ \\
\hline $\mathrm{C} 5-\mathrm{H} 5$ & 0.9500 & $\mathrm{C} 13-\mathrm{H} 13 \mathrm{~A}$ & 0.9800 \\
\hline $\mathrm{C} 6-\mathrm{C} 7$ & $1.500(4)$ & $\mathrm{C} 13-\mathrm{H} 13 \mathrm{~B}$ & 0.9800 \\
\hline C6-H6A & 0.9900 & $\mathrm{C} 13-\mathrm{H} 13 \mathrm{C}$ & 0.9800 \\
\hline C6-H6B & 0.9900 & $\mathrm{~N} 5-\mathrm{C} 14$ & $1.110(5)$ \\
\hline $\mathrm{C} 7-\mathrm{C} 8$ & $1.383(4)$ & $\mathrm{C} 14-\mathrm{C} 15$ & $1.445(5)$ \\
\hline $\mathrm{C} 7-\mathrm{C} 11$ & $1.387(4)$ & $\mathrm{C} 15-\mathrm{H} 15 \mathrm{~A}$ & 0.9800 \\
\hline $\mathrm{C} 8-\mathrm{H} 8$ & 0.9500 & C15-H15B & 0.9800 \\
\hline $\mathrm{C} 9-\mathrm{C} 10$ & $1.375(4)$ & $\mathrm{C} 15-\mathrm{H} 15 \mathrm{C}$ & 0.9800 \\
\hline $\mathrm{N} 1{ }^{\mathrm{i}}-\mathrm{Ag} 1-\mathrm{N} 2$ & $170.55(8)$ & $\mathrm{C} 10-\mathrm{C} 11-\mathrm{H} 11$ & 120.5 \\
\hline $\mathrm{N} 1^{\mathrm{i}}-\mathrm{Ag} 1-\mathrm{Ag} 1^{\mathrm{ii}}$ & $100.46(6)$ & $\mathrm{C} 7-\mathrm{C} 11-\mathrm{H} 11$ & 120.5 \\
\hline $\mathrm{N} 2-\mathrm{Ag} 1-\mathrm{Ag} 1^{\mathrm{ii}}$ & $84.17(6)$ & $\mathrm{F} 3^{\prime}-\mathrm{P} 1-\mathrm{F} 1^{\prime}$ & $98.8(9)$ \\
\hline $\mathrm{C} 1-\mathrm{N} 1-\mathrm{C} 5$ & $119.3(2)$ & $\mathrm{F} 5-\mathrm{P} 1-\mathrm{F} 6$ & $96.7(5)$ \\
\hline $\mathrm{C} 1-\mathrm{N} 1-\mathrm{Ag} 1^{\mathrm{iii}}$ & $119.39(18)$ & $\mathrm{F} 3^{\prime}-\mathrm{P} 1-\mathrm{F} 4^{\prime}$ & $93.7(10)$ \\
\hline $\mathrm{C} 5-\mathrm{N} 1-\mathrm{Ag} 1^{1 \mathrm{iii}}$ & $121.31(17)$ & $\mathrm{F} 1^{\prime}-\mathrm{P} 1-\mathrm{F} 4^{\prime}$ & $86.1(10)$ \\
\hline $\mathrm{C} 8-\mathrm{N} 2-\mathrm{C} 9$ & $117.9(2)$ & $\mathrm{F} 5-\mathrm{P} 1-\mathrm{F} 4$ & $90.8(9)$ \\
\hline $\mathrm{C} 8-\mathrm{N} 2-\mathrm{Ag} 1$ & $121.18(18)$ & $\mathrm{F} 6-\mathrm{P} 1-\mathrm{F} 4$ & $92.8(7)$ \\
\hline $\mathrm{C} 9-\mathrm{N} 2-\mathrm{Ag} 1$ & $120.91(17)$ & $\mathrm{F} 5-\mathrm{P} 1-\mathrm{F} 2$ & $94.0(5)$ \\
\hline $\mathrm{C} 2-\mathrm{N} 3-\mathrm{C} 6$ & $121.4(2)$ & $\mathrm{F} 6-\mathrm{P} 1-\mathrm{F} 2$ & $90.7(5)$ \\
\hline $\mathrm{C} 2-\mathrm{N} 3-\mathrm{H} 3 \mathrm{~A}$ & $117(2)$ & $\mathrm{F} 4-\mathrm{P} 1-\mathrm{F} 2$ & $173.7(8)$ \\
\hline $\mathrm{C} 6-\mathrm{N} 3-\mathrm{H} 3 \mathrm{~A}$ & $121(2)$ & $\mathrm{F} 3^{\prime}-\mathrm{P} 1-\mathrm{F} 2^{\prime}$ & $96.2(8)$ \\
\hline $\mathrm{N} 1-\mathrm{C} 1-\mathrm{C} 2$ & $122.6(2)$ & $\mathrm{F} 1^{\prime}-\mathrm{P} 1-\mathrm{F} 2^{\prime}$ & $92.7(6)$ \\
\hline
\end{tabular}




\begin{tabular}{|c|c|c|c|}
\hline $\mathrm{N} 1-\mathrm{C} 1-\mathrm{H} 1$ & 118.7 & $\mathrm{~F} 4^{\prime}-\mathrm{P} 1-\mathrm{F} 2^{\prime}$ & $170.1(8)$ \\
\hline $\mathrm{C} 2-\mathrm{C} 1-\mathrm{H} 1$ & 118.7 & $\mathrm{~F} 5-\mathrm{P} 1-\mathrm{F} 3$ & $91.0(4)$ \\
\hline $\mathrm{N} 3-\mathrm{C} 2-\mathrm{C} 3$ & $122.6(2)$ & $\mathrm{F} 6-\mathrm{P} 1-\mathrm{F} 3$ & $172.3(4)$ \\
\hline $\mathrm{N} 3-\mathrm{C} 2-\mathrm{C} 1$ & $119.6(2)$ & $\mathrm{F} 4-\mathrm{P} 1-\mathrm{F} 3$ & $86.5(6)$ \\
\hline $\mathrm{C} 3-\mathrm{C} 2-\mathrm{C} 1$ & $117.8(2)$ & $\mathrm{F} 2-\mathrm{P} 1-\mathrm{F} 3$ & $89.3(5)$ \\
\hline $\mathrm{C} 4-\mathrm{C} 3-\mathrm{C} 2$ & $118.8(2)$ & $\mathrm{F} 3^{\prime}-\mathrm{P} 1-\mathrm{F} 5^{\prime}$ & $88.8(8)$ \\
\hline $\mathrm{C} 4-\mathrm{C} 3-\mathrm{H} 3$ & 120.6 & $\mathrm{~F} 1^{\prime}-\mathrm{P} 1-\mathrm{F} 5^{\prime}$ & $172.5(8)$ \\
\hline $\mathrm{C} 2-\mathrm{C} 3-\mathrm{H} 3$ & 120.6 & $\mathrm{~F} 4^{\prime}-\mathrm{P} 1-\mathrm{F} 5^{\prime}$ & $93.3(11)$ \\
\hline $\mathrm{C} 5-\mathrm{C} 4-\mathrm{C} 3$ & 120.0 & $\mathrm{~F} 2^{\prime}-\mathrm{P} 1-\mathrm{F} 5^{\prime}$ & $86.6(6)$ \\
\hline $\mathrm{C} 5-\mathrm{C} 4-\mathrm{H} 4$ & 120.0 & $\mathrm{~F} 5-\mathrm{P} 1-\mathrm{F} 1$ & $173.5(4)$ \\
\hline $\mathrm{C} 3-\mathrm{C} 4-\mathrm{H} 4$ & 120.0 & $\mathrm{~F} 6-\mathrm{P} 1-\mathrm{F} 1$ & $89.4(4)$ \\
\hline $\mathrm{N} 1-\mathrm{C} 5-\mathrm{C} 4$ & $121.5(2)$ & $\mathrm{F} 4-\mathrm{P} 1-\mathrm{F} 1$ & $91.3(9)$ \\
\hline $\mathrm{N} 1-\mathrm{C} 5-\mathrm{H} 5$ & 119.2 & $\mathrm{~F} 2-\mathrm{P} 1-\mathrm{F} 1$ & $83.5(4)$ \\
\hline $\mathrm{C} 4-\mathrm{C} 5-\mathrm{H} 5$ & 119.2 & $\mathrm{~F} 3-\mathrm{P} 1-\mathrm{F} 1$ & $82.9(3)$ \\
\hline $\mathrm{N} 3-\mathrm{C} 6-\mathrm{C} 7$ & $111.4(2)$ & $\mathrm{F} 3^{\prime}-\mathrm{P} 1-\mathrm{F} 6^{\prime}$ & $171.5(8)$ \\
\hline $\mathrm{N} 3-\mathrm{C} 6-\mathrm{H} 6 \mathrm{~A}$ & 109.3 & $\mathrm{~F} 1^{\prime}-\mathrm{P} 1-\mathrm{F} 6^{\prime}$ & $89.8(8)$ \\
\hline $\mathrm{C} 7-\mathrm{C} 6-\mathrm{H} 6 \mathrm{~A}$ & 109.3 & $\mathrm{~F} 4^{\prime}-\mathrm{P} 1-\mathrm{F} 6^{\prime}$ & $87.3(8)$ \\
\hline N3-C6- $-\mathrm{H} 6 \mathrm{~B}$ & 109.3 & $\mathrm{~F} 2^{\prime}-\mathrm{P} 1-\mathrm{F} 6^{\prime}$ & $82.9(5)$ \\
\hline $\mathrm{C} 7-\mathrm{C} 6-\mathrm{H} 6 \mathrm{~B}$ & 109.3 & $\mathrm{~F} 5^{\prime}-\mathrm{P} 1-\mathrm{F} 6^{\prime}$ & $82.7(5)$ \\
\hline $\mathrm{H} 6 \mathrm{~A}-\mathrm{C} 6-\mathrm{H} 6 \mathrm{~B}$ & 108.0 & $\mathrm{~N} 4-\mathrm{C} 12-\mathrm{C} 13$ & $179.6(5)$ \\
\hline $\mathrm{C} 8-\mathrm{C} 7-\mathrm{C} 11$ & $118.0(2)$ & $\mathrm{C} 12-\mathrm{C} 13-\mathrm{H} 13 \mathrm{~A}$ & 109.5 \\
\hline $\mathrm{C} 8-\mathrm{C} 7-\mathrm{C} 6$ & $120.1(3)$ & $\mathrm{C} 12-\mathrm{C} 13-\mathrm{H} 13 \mathrm{~B}$ & 109.5 \\
\hline $\mathrm{C} 11-\mathrm{C} 7-\mathrm{C} 6$ & $121.9(2)$ & $\mathrm{H} 13 \mathrm{~A}-\mathrm{C} 13-\mathrm{H} 13 \mathrm{~B}$ & 109.5 \\
\hline $\mathrm{N} 2-\mathrm{C} 8-\mathrm{C} 7$ & $123.4(2)$ & $\mathrm{C} 12-\mathrm{C} 13-\mathrm{H} 13 \mathrm{C}$ & 109.5 \\
\hline $\mathrm{N} 2-\mathrm{C} 8-\mathrm{H} 8$ & 118.3 & $\mathrm{H} 13 \mathrm{~A}-\mathrm{C} 13-\mathrm{H} 13 \mathrm{C}$ & 109.5 \\
\hline $\mathrm{C} 7-\mathrm{C} 8-\mathrm{H} 8$ & 118.3 & $\mathrm{H} 13 \mathrm{~B}-\mathrm{C} 13-\mathrm{H} 13 \mathrm{C}$ & 109.5 \\
\hline $\mathrm{N} 2-\mathrm{C} 9-\mathrm{C} 10$ & $122.4(3)$ & $\mathrm{N} 5-\mathrm{C} 14-\mathrm{C} 15$ & $177.5(4)$ \\
\hline $\mathrm{N} 2-\mathrm{C} 9-\mathrm{H} 9$ & 118.8 & $\mathrm{C} 14-\mathrm{C} 15-\mathrm{H} 15 \mathrm{~A}$ & 109.5 \\
\hline $\mathrm{C} 10-\mathrm{C} 9-\mathrm{H} 9$ & 118.8 & $\mathrm{C} 14-\mathrm{C} 15-\mathrm{H} 15 \mathrm{~B}$ & 109.5 \\
\hline $\mathrm{C} 9-\mathrm{C} 10-\mathrm{C} 11$ & $119.3(3)$ & $\mathrm{H} 15 \mathrm{~A}-\mathrm{C} 15-\mathrm{H} 15 \mathrm{~B}$ & 109.5 \\
\hline $\mathrm{C} 9-\mathrm{C} 10-\mathrm{H} 10$ & 120.3 & $\mathrm{C} 14-\mathrm{C} 15-\mathrm{H} 15 \mathrm{C}$ & 109.5 \\
\hline $\mathrm{C} 11-\mathrm{C} 10-\mathrm{H} 10$ & 120.3 & $\mathrm{H} 15 \mathrm{~A}-\mathrm{C} 15-\mathrm{H} 15 \mathrm{C}$ & 109.5 \\
\hline $\mathrm{C} 10-\mathrm{C} 11-\mathrm{C} 7$ & $119.0(3)$ & $\mathrm{H} 15 \mathrm{~B}-\mathrm{C} 15-\mathrm{H} 15 \mathrm{C}$ & 109.5 \\
\hline $\mathrm{C} 5-\mathrm{N} 1-\mathrm{C} 1-\mathrm{C} 2$ & $0.5(4)$ & $\mathrm{N} 3-\mathrm{C} 6-\mathrm{C} 7-\mathrm{C} 8$ & $-102.6(3)$ \\
\hline $\mathrm{Ag} 1{ }^{\mathrm{iii}}-\mathrm{N} 1-\mathrm{C} 1-\mathrm{C} 2$ & $-179.21(19)$ & $\mathrm{N} 3-\mathrm{C} 6-\mathrm{C} 7-\mathrm{C} 11$ & $79.0(3)$ \\
\hline $\mathrm{C} 6-\mathrm{N} 3-\mathrm{C} 2-\mathrm{C} 3$ & $-6.3(4)$ & $\mathrm{C} 9-\mathrm{N} 2-\mathrm{C} 8-\mathrm{C} 7$ & $-0.1(4)$ \\
\hline $\mathrm{C} 6-\mathrm{N} 3-\mathrm{C} 2-\mathrm{C} 1$ & $176.2(3)$ & $\mathrm{Ag} 1-\mathrm{N} 2-\mathrm{C} 8-\mathrm{C} 7$ & $178.53(19)$ \\
\hline $\mathrm{N} 1-\mathrm{C} 1-\mathrm{C} 2-\mathrm{N} 3$ & $176.7(3)$ & $\mathrm{C} 11-\mathrm{C} 7-\mathrm{C} 8-\mathrm{N} 2$ & $-0.6(4)$ \\
\hline $\mathrm{N} 1-\mathrm{C} 1-\mathrm{C} 2-\mathrm{C} 3$ & $-0.9(4)$ & $\mathrm{C} 6-\mathrm{C} 7-\mathrm{C} 8-\mathrm{N} 2$ & $-179.0(2)$ \\
\hline $\mathrm{N} 3-\mathrm{C} 2-\mathrm{C} 3-\mathrm{C} 4$ & $-176.8(3)$ & $\mathrm{C} 8-\mathrm{N} 2-\mathrm{C} 9-\mathrm{C} 10$ & $0.3(4)$ \\
\hline $\mathrm{C} 1-\mathrm{C} 2-\mathrm{C} 3-\mathrm{C} 4$ & $0.7(4)$ & $\mathrm{Ag} 1-\mathrm{N} 2-\mathrm{C} 9-\mathrm{C} 10$ & $-178.3(2)$ \\
\hline $\mathrm{C} 2-\mathrm{C} 3-\mathrm{C} 4-\mathrm{C} 5$ & $-0.3(4)$ & $\mathrm{N} 2-\mathrm{C} 9-\mathrm{C} 10-\mathrm{C} 11$ & $0.1(4)$ \\
\hline $\mathrm{C} 1-\mathrm{N} 1-\mathrm{C} 5-\mathrm{C} 4$ & $0.0(4)$ & $\mathrm{C} 9-\mathrm{C} 10-\mathrm{C} 11-\mathrm{C} 7$ & $-0.8(4)$ \\
\hline $\mathrm{Ag} 1{ }^{\mathrm{iii}-\mathrm{N}} 1-\mathrm{C} 5-\mathrm{C} 4$ & $179.7(2)$ & $\mathrm{C} 8-\mathrm{C} 7-\mathrm{C} 11-\mathrm{C} 10$ & $1.0(4)$ \\
\hline
\end{tabular}


$\mathrm{C} 3-\mathrm{C} 4-\mathrm{C} 5-\mathrm{N} 1$

$\mathrm{C} 2-\mathrm{N} 3-\mathrm{C} 6-\mathrm{C} 7$
-0.1 (4)

$174.7(3)$
$\mathrm{C} 6-\mathrm{C} 7-\mathrm{C} 11-\mathrm{C} 10$

$179.4(3)$

Symmetry codes: (i) $x+1 / 2,-y+1 / 2, z+1 / 2$; (ii) $-x+1,-y+1,-z+1$; (iii) $x-1 / 2,-y+1 / 2, z-1 / 2$.

Hydrogen-bond geometry $\left(\AA,{ }^{\circ}\right)$

\begin{tabular}{|c|c|c|c|c|}
\hline$D-\mathrm{H} \cdots A$ & $D-\mathrm{H}$ & $\mathrm{H} \cdots A$ & $D \cdots A$ & $D-\mathrm{H} \cdots A$ \\
\hline $\mathrm{N} 3-\mathrm{H} 3 A \cdots \mathrm{F} 3$ & $0.84(3)$ & $2.49(3)$ & $3.208(8)$ & $144(3)$ \\
\hline $\mathrm{N} 3-\mathrm{H} 3 A \cdots \mathrm{F} 5$ & $0.84(3)$ & $2.46(3)$ & $3.273(10)$ & $162(3)$ \\
\hline $\mathrm{N} 3-\mathrm{H} 3 A \cdots \mathrm{F} 5^{\prime}$ & $0.84(3)$ & $2.18(3)$ & $2.999(10)$ & $167(3)$ \\
\hline $\mathrm{C} 8-\mathrm{H} 8 \cdots \mathrm{N} 4^{\mathrm{ii}}$ & 0.95 & 2.63 & $3.478(5)$ & 149 \\
\hline $\mathrm{C} 10-\mathrm{H} 10 \cdots \mathrm{F} 2^{\mathrm{iv}}$ & 0.95 & 2.39 & $3.176(9)$ & 140 \\
\hline $\mathrm{C} 13-\mathrm{H} 13 A \cdots \mathrm{F} 2^{\prime \text { iv }}$ & 0.98 & 2.54 & $3.487(13)$ & 163 \\
\hline $\mathrm{C} 13-\mathrm{H} 13 B^{\cdots} \cdots \mathrm{F}^{\mathrm{i}}$ & 0.98 & 2.53 & $3.365(10)$ & 144 \\
\hline $\mathrm{C} 13-\mathrm{H} 13 B \cdots \mathrm{F} 1^{\prime \mathrm{i}}$ & 0.98 & 2.59 & $3.57(2)$ & 171 \\
\hline $\mathrm{C} 15-\mathrm{H} 15 A \cdots \mathrm{F} 1$ & 0.98 & 2.52 & $3.441(9)$ & 157 \\
\hline $\mathrm{C} 15-\mathrm{H} 15 A \cdots \mathrm{F} 3^{\prime}$ & 0.98 & 2.42 & $3.356(13)$ & 160 \\
\hline $\mathrm{C} 15-\mathrm{H} 15 B \cdots \mathrm{F} 2^{\mathrm{v}}$ & 0.98 & 2.23 & $3.088(10)$ & 146 \\
\hline $\mathrm{C} 15-\mathrm{H} 15 B \cdots \mathrm{F} 5^{\prime v}$ & 0.98 & 2.57 & $3.509(11)$ & 160 \\
\hline $\mathrm{C} 15-\mathrm{H} 15 C \cdots \mathrm{F} 1^{\mathrm{iv}}$ & 0.98 & 2.36 & $3.329(8)$ & 168 \\
\hline $\mathrm{C} 15-\mathrm{H} 15 C \cdots \mathrm{F} 1^{\text {,iv }}$ & 0.98 & 2.09 & $3.037(9)$ & 161 \\
\hline
\end{tabular}

Symmetry codes: (i) $x+1 / 2,-y+1 / 2, z+1 / 2$; (ii) $-x+1,-y+1,-z+1$; (iv) $-x+3 / 2, y+1 / 2,-z+1 / 2$; (v) $-x+3 / 2, y-1 / 2,-z+1 / 2$. 